\title{
La Visión Institucional de la Geopolítica de los Recursos Hídricos en Venezuela (Estado, Nación y Gobierno)
}

Autor: Carlos Javier Lizcano Chapeta Universidad de Los Andes, ULA lizcha 4@hotmail.com Mérida, Venezuela

\section{Resumen}

En el contexto mundial, regional y local los recursos hídricos constituyen un elemento estratégico desde el punto de vista geopolítico, dado la escasez del mismo y el manejo que debe darse a dicha problemática desde los Estados, gobiernos y naciones. En este sentido, el propósito es analizar la importancia estratégica que se ha dado a los recursos hídricos en Venezuela, teniendo en cuenta la visión del Estado, gobierno y nación. Se utiliza un diseño documental, de tipo descriptivo, y como técnica de recolección de datos se emplea el arqueo bibliográfico y el análisis de contenido de fuentes anteriores. Los resultados señalan que el debate internacional sobre la importancia de los recursos hídricos es un hecho y que en países como Venezuela que poseen grandes reservas se debe adoptar una estrategia que vaya dirigida a una verdadera gestión integral de los recursos hídricos. Se concluye que es urgente el trabajo coordinado entre el gobierno nacional y los gobiernos locales para hacer cumplir las normativas creadas por el Estado y operacionalizar con las instituciones que se han establecido para la gestión del recurso agua en el país.

Palabras clave: recursos hídricos; geopolítica; Venezuela; estado; nación; gobierno. 


\title{
The Institutional Vision of the Geopolitics of Water Resources in Venezuela (State, Nation and Government)
}

\begin{abstract}
In the global, regional and local context, water resources are a strategic element from the geopolitical point of view, given the scarcity of water and the management that must be given to this problem from States, governments and nations. In this sense, the purpose is to analyze the strategic importance that has been given to water resources in Venezuela, taking into account the vision of the State, government and nation. A documentary design is used, of descriptive type, and as a data collection technique, bibliographic archiving and content analysis of previous sources are used. The results indicate that the international debate on the importance of water resources is a fact and that in countries such as Venezuela that have great reserves, a strategy must be adopted that aims at a true integral management of water resources. It is concluded that it is urgent the coordinated work between the national government and local governments to enforce the regulations created by the State and operationalize with the institutions that have been established for the management of water resources in the country.
\end{abstract}

Keywords: water resources; geopolitics; Venezuela; state; nation; government.

Date Received: 15-02-2017

Date Acceptance: 17-03-2017 


\section{Introducción}

Existe una problemática de escasez de agua dulce y por ende es imprescindible la preservación de los recursos hídricos, se requiere de una nueva visión geopolítica que haga valer las directrices internacionales y la soberanía entorno a ciertas áreas geográficas estratégicas de América del sur que hoy poseen grandes reservas de agua dulce. En la actualidad en el concierto de las relaciones internacionales el acceso a los recursos naturales, las materias primas y los recursos energéticos constituyen interés vital para los países del mundo.

Dentro de estos territorios que poseen grandes reservas de agua se encuentra Venezuela con una posición geográfica por excelencia, con una red hidrográfica que le permite conectarse con el Caribe y el Atlántico. En este sentido, De Lisio (2008), sostiene la relevancia de Venezuela por su localización en la zona ecuatorial, como punto de contacto geológico entre las placas tectónicas de América del Sur y del Caribe. Y por la encrucijada ecogeográfica entre los grandes sistemas físico-bióticos-culturales de importancia regional y mundial: el Caribe, los Andes, la Orinoquia y el Amazonas.

Igualmente se cuenta con recursos hídricos subterráneos, los llamados acuíferos y los embalses para generar energía eléctrica, como el Gurí. Según el informe de la Comisión Permanente de Ambiente, Recursos Naturales y Cambio Climático de la Asamblea Nacional (2016), los acuíferos se clasifican en acuíferos de gran potencialidad: se ubican en la Mesa de Guanipa del estado Anzoátegui, sur de Monagas, sistema de riego del río Guárico, llanos de Barinas, llanos de Portuguesa y llanos de Apure. Acuíferos con potencial medio: Barlovento, Valle de Caracas. Y los acuíferos en vía de agotamiento: Valle de Quibor, Nirgua, Coro, Margarita, entre otros. El mismo informe señala que existen alrededor de 110 embalses distribuidos a lo largo del territorio nacional, construidos para satisfacer fines diversos entre los que destacan el consumo humano y la generación de energía. 
Ahora bien, desde el punto de vista histórico, Venezuela es un país que cuenta con tradición en el manejo de los recursos hídricos, según informe del Ministerio del Ambiente (1998), durante la Capitanía General, particularmente en el siglo XVIII se realizaron o se propusieron importantes obras para el manejo racional de sus aguas. Los principios de su administración hidráulica derivan de los usos y costumbres trasladados de la península ibérica, resultado de las experiencias de los pueblos que la habitaron y que debieron adaptarse a sus condiciones hidrográficas, particularmente las del árido sur.

Dicho lo anterior, el propósito de la investigación fue examinar las distintas posiciones y acciones que desde el Estado, la nación y el gobierno venezolano se han desarrollado para abordar la problemática de la escasez de agua dulce, dada la importancia estratégica que este recurso natural ha tomado en los contextos actuales de la geopolítica latinoamericana y mundial.

\section{Fundamentación teórica}

Es importante distinguir algunos conceptos propios del tema que permitan abordar con precisión el papel que el Estado, la nación y el gobierno han desempeñado en función de generar una visión actualizada y estratégica, en la preservación y manejo de los recursos hídricos en el país.

\subsection{Una mirada desde el Estado}

El Estado es una organización institucional, política y jurídica que se caracteriza por una serie de elementos constitutivos, entre los que se destacan, la soberanía, el territorio, la población, un aparato administrativo de naturaleza burocrática y un gobierno. Ahora bien, corresponde al Estado a través de su andamiaje institucional y jurídico la implementación de medidas para abordar la situación problemática del agua en Venezuela. 


\subsubsection{La función de los organismos internacionales}

La preocupación por atender las problemáticas ambientales se ha generado desde los organismos internacionales, los cuales han establecido las orientaciones y mecanismos generales para que sean adoptados en los diferentes países. De Lisio (2008), propone el llamado Estado multidimensional, el cual "va surgiendo a raíz de los problemas ambientales del mundo y la necesidad de ir más allá de las fronteras de los propios Estados para abordar una problemática cuya responsabilidad no puede involucrar a un sólo país" (pág. 53).

El año de 1992 marca un hito en la historia para abordar la problemática ambiental, a través de los organismos internacionales se da la Conferencia Internacional sobre Medio Ambiente y Desarrollo realizada en Río de Janeiro. La llamada agenda 21, propone en cuanto a la conservación y gestión de los recursos lo siguiente: "protección de la atmósfera; lucha contra la deforestación; lucha contra desertificación; desarrollo sostenible de las áreas de montaña; conservación de la diversidad biológica; protección y gestión de los océanos; protección y gestión de los recursos de agua dulce" (pág. 36).

En 1996 se constituyen dos organizaciones globales en torno al agua: El Consejo Mundial del Agua y La Asociación Mundial del Agua. El propósito de estas organizaciones está orientado a la gestión de los recursos hídricos, generando directrices para los países desarrollados y en desarrollo, agencias de Naciones Unidas, organizaciones no gubernamentales del sector privado. Por otro lado, en 1997 los gobiernos acordaron ampliar el Convenio Marco sobre el Cambio Climático (CMCC) mediante la suscripción del Protocolo de Kyoto, el cual entró en vigor en febrero de 2005, cuando 55 países responsables de la emisión de gases de efecto invernadero accedieron a firmarlo.

Entonces, el siglo XXI va a estar marcado por el compromiso de los países del mundo en atender las problemáticas ambientales, dando 
cumplimiento a las disposiciones de las conferencias internacionales que desde la época se vienen realizando, específicamente aquellas relacionadas con el agua dulce su preservación y gestión.

\subsubsection{El Estado venezolano}

La institucionalidad en materia de agua y problemáticas ambientales en Venezuela, comienza según De Lisio (ob.cit), a mediados de los años setenta cuando se crea el Ministerio del Ambiente y los Recursos Naturales Renovables (MARNR), sobre la base de los postulados del Ecodesarrollo, fue una respuesta a las exigencias de los grupos ciudadanos agrupados en organizaciones de acción y presión ambiental.

De acuerdo con el informe de la Red ARA (2011), Venezuela es reconocida en el ámbito latinoamericano como país pionero y ejemplo en temas de normativa legal, conservación de áreas de importancia ecológica y programas de gestión de recursos naturales. El Estado ha suscrito más de treinta Convenios Internacionales, que en forma de Leyes Aprobatorias, obligan al Estado venezolano a actuar efectivamente en la conservación de las condiciones ambientales y en la defensa del patrimonio ambiental común.

El mismo informe establece que Venezuela alcanzó las metas del milenio en cuanto a la cobertura de agua potable (2001) y de recolección de aguas servidas (2005), pero aún hay desafíos. El 95\% de los venezolanos tienen acceso al agua potable (INE, 2011), pero muchas zonas aún no tienen un suministro permanente.

Ahora bien, en cuanto a la normativa legal, el Estado venezolano ha venido tratando el tema ambiental, partiendo de la Constitución de la República Bolivariana de Venezuela que en su artículo 127 establece el derecho de todos sus ciudadanos a un ambiente sano, seguro y ecológicamente equilibrado. Así mismo, determina la protección del recurso agua como obligatoriedad del Estado junto con la participación de la sociedad. 
Constitucionalmente se coloca a la sociedad como uno de los actores prioritarios en la gestión ambiental. Este principio se reafirma en la Ley Orgánica del Ambiente al establecer la corresponsabilidad, participación y tutela efectiva de la sociedad, en su artículo 4. Otro instrumento jurídico de gran importancia, es la Ley de Aguas, al considerar el agua como un derecho humano fundamental, le otorga un carácter estratégico para el país e incorpora importantes conceptos como la gestión integral de las aguas, el bienestar humano y el desarrollo sustentable. En el siguiente cuadro se realiza una presentación de los diferentes instrumentos jurídicos establecidos por el Estado venezolano y que representa una fortaleza para el sector, ya que de allí se desprenden lineamientos para el manejo integral de los recursos hídricos.

Cuadro № 1. Ordenamiento jurídico del Estado venezolano en el tema agua.

\begin{tabular}{|c|l|}
\hline Instrumento Jurídico & \multicolumn{1}{|c|}{ Aspecto que trata } \\
\hline Constitución Nacional & $\begin{array}{l}\text { El agua como bien público. Protección } \\
\text { del agua. }\end{array}$ \\
\hline Ley Orgánica del Ambiente & $\begin{array}{l}\text { Gestión integral del agua para } \\
\text { garantizar la calidad, cantidad y } \\
\text { disponibilidad del agua para sustentar } \\
\text { el ciclo hidrológico. Protección de las } \\
\text { cuencas hidrográficas. }\end{array}$ \\
\hline Ley de Aguas & $\begin{array}{l}\text { Acceso al agua como derecho } \\
\text { humano fundamental. El agua como } \\
\text { un bien social y como un recurso } \\
\text { insustituible para la vida y para el } \\
\text { desarrollo social y económico. }\end{array}$ \\
\hline Ley de Tierras y Desarrollo & $\begin{array}{l}\text { Censo de aguas para el uso agrario. } \\
\text { Agrario }\end{array}$ \\
\hline $\begin{array}{c}\text { Ley Orgánica para la Prestación } \\
\text { de los Servicios de Agua Potable } \\
\text { y de Saneamiento }\end{array}$ & $\begin{array}{l}\text { Fiscalización, control y evaluación de } \\
\text { los servicios de agua potable y de } \\
\text { saneamiento. }\end{array}$ \\
\hline Ley de Régimen Municipal & $\begin{array}{l}\text { Competencia municipal para la } \\
\text { prestación del servicio de }\end{array}$ \\
\hline
\end{tabular}




\begin{tabular}{|c|l|}
\hline & $\begin{array}{l}\text { abastecimiento domiciliario de agua } \\
\text { potable. }\end{array}$ \\
\hline $\begin{array}{c}\text { Ley de Gestión Integral de } \\
\text { Riesgos Socionaturales y } \\
\text { Tecnológicos }\end{array}$ & $\begin{array}{l}\text { Armonizar competencias nacionales, } \\
\text { estadales y municipales para prevenir } \\
\text { riesgos en una localidad tomando en } \\
\text { cuenta realidades ecológicas. }\end{array}$ \\
\hline
\end{tabular}

Fuente: Informe Asamblea Nacional (2016).

La institucionalidad asociada a la administración del agua se rige por leyes distintas. En el caso del agua como recurso, la Ley de Aguas (2007), establece en el artículo 21, la siguiente organización: El Ministerio con competencia para dicha materia, quien ejerce la Autoridad Nacional de las Aguas. El Consejo Nacional de las Aguas. Los Consejos de Región Hidrográfica. Los Consejos de Cuencas Hidrográficas. Los usuarios o las usuarias institucionales. Los Consejos Comunales. Las Mesas Técnicas y Comités de Riego. El Instituto Nacional de Pueblos Indígenas. El Ministerio con competencia en materia de Defensa, a través del componente correspondiente. Los Consejos Estadales de Planificación y Coordinación de Políticas Públicas. Los Consejos Locales de Planificación Pública.

Esta estructura organizacional debe trabajar coordinadamente, con las Empresas Hidrológicas, filiales de HIDROVEN y Descentralizadas, para contribuir con una mejor administración y manejo del recurso hídrico. Igualmente, es importante el rol de algunas instituciones del Estado creadas recientemente como son: El Ministerio de Ecosocialismo y Aguas; la Oficina Nacional para el Desarrollo de los Servicios de Agua Potable y de Saneamiento; la Superintendencia Nacional de los Servicios de Agua Potable y de Saneamiento; y la Empresa de Gestión Nacional. Ahora bien, el Ejecutivo Nacional, consciente de la problemática del agua, creó también el Consejo Presidencial del Agua y/o el Estado Mayor del Agua, que se suman al conjunto 
de Órganos Superiores Sectoriales y/o Estados Mayores por área, que se han constituido en la actual administración.

En tal sentido, el Estado venezolano ha venido creando todo un entramado jurídico e institucional que hasta la presente fecha no ha podido dar respuesta al problema de la escasez y gestión integral de los recursos hídricos en el país, así se evidencia del informe de la Asamblea Nacional (2016), a través de la comisión permanente de ambiente, recursos naturales y cambio climático, el cual señala entre otras cosas: "la institucionalidad del sector es una trama de instituciones cuyas relaciones con HIDROVEN, sus empresas filiales y empresas descentralizadas no resulta clara, y dificulta la labor de coordinación de planes e identificación de responsabilidades". (pág. 52).

\subsection{La visión holística y compleja desde la Nación}

Cuando se habla de una visión holística y compleja de la nación, se quieren retomar las ideas de Rojas (2011), el cual revisa nuevos enfoques y problemas que tienen que ver con la historia de la nación, que deben ser abordados desde la interdisciplinariedad, bajo una visión holística y compleja. La dinámica actual con su aceleración de la historia, el desconocimiento del pasado, la vivencia de los tiempos efímeros tan presentes en la postmodernidad, parecen estar desplazando a las sociedades con memoria, que transmitían valores a través de instituciones como la escuela, la familia, la iglesia o el Estado.

Para este investigador el tema nación y nacionalismos se plantean como conceptos vivos y cambiantes expresados en las acciones y el discurso variado y no pocas veces contradictorio en estos inicios del siglo XXI, donde el nacionalismo puede expresar desde posiciones antiimperialistas hasta fundamentalismos religiosos y exaltación de diferencias étnicas y culturales, todo ello enmarcado en un mundo cada vez más globalizado. 
De manera que abordar en la actualidad el estudio de los problemas ambientales y específicamente la escasez de agua dulce en el mundo y en particular en Venezuela, así como su preservación y gestión integral, requiere de esa óptica amplia, incluyente que tome en cuenta las posiciones de las diferentes culturas. Al respecto Quagliotti (2011), explica que el tema del agua como recurso vital de las civilizaciones conlleva hacía una revalorización geopolítica y geoeconómica de ciertos lugares, acompañada de sus dimensiones culturales, sociales y políticas.

En consecuencia, la cultura hacía el tratamiento del agua dulce, es un tema prioritario a favor de la continuidad de la vida de la humanidad, sostiene Quagliotti (ob.cit). El gran conflicto radica en cuanto a la escasez de conciencia sobre el tema, el cual provoca en muchas regiones graves perjuicios en relación a la preservación, mantenimiento, gestión y distribución de los recursos hídricos.

En lo que respecta a la nación venezolana, de acuerdo a estudios realizados por la fundación Vitalis (2013), lamentablemente no se ha desarrollado una cultura hacia la preservación, cuidado y gestión del agua dulce en nuestras sociedades, pese a las situaciones fuertes de escasez del recurso y de energía eléctrica que hemos padecido en los últimos años.

Esta situación ha generado, según el informe de la fundación, falta de conciencia en la población, al hacer un uso desmedido del recurso hídrico y de la energía eléctrica, sin ningún tipo de control; las campañas de publicidad no han dado los mejores frutos. Finalmente, la educación ambiental no produce los efectos esperados para cambiar en la población las viejas costumbres y crear nuevos comportamientos en torno a mantener y preservar los recursos naturales, como el agua. 
Se requiere entonces, de educación y conciencia ambiental que está basada según Vitalis (ob.cit), en los siguientes aspectos:

Campañas de sensibilización e información ambiental que contribuyan a valorar el agua y la energía. Promover la participación ciudadana a fin de fortalecer la contraloría social de la gestión ambiental, donde se estimule y premie a los funcionarios capaces y responsables, se penalice a los culpables y se documenten las infracciones (pág. 29).

También es importante el papel de las diferentes culturas étnicas en torno al problema del agua, es preciso valorar la relación del hombre con la naturaleza, ello implica hacerlo desde una perspectiva holística que dé cuenta de nuestro reconocimiento como "hijos de la tierra, hijos de la vida, hijos del cosmos. Nos haría tomar conciencia de nuestra comunidad de destino, amenazados por los mismos peligros mortales. Sabríamos, que el pequeño planeta perdido llamado tierra es nuestra casa, es nuestra tierra-patria" (Morin, 2011: pág. 10).

En un mundo complejo, con problemas complejos adquiere especial relevancia comprometerse con el rescate de los valores hacía el cuidado y la preservación de los recursos de la madre tierra, especialmente el agua dulce, dada la grave situación de su escasez. Es por ello, que Morin (ob.cit), señala: "la toma de conciencia de esa comunidad de destino debe convertirse en el acontecimiento clave del siglo XXI: debemos sentirnos solidarios con este planeta cuya vida condiciona la nuestra. Hay que salvar nuestra Pachamama" (pág. 86).

Se debe tomar en cuenta la revisión de las creencias, concepciones, actitudes y valores que predominan en algunos grupos étnicos del país, con el firme propósito de que sean aceptadas en pro de la utilización y preservación de los recursos hídricos. Es bien cierto, como señala Boaventura de Sousa (2010), que en distintas áreas de la vida social la ciencia ha demostrado tener una superioridad innegable, sin embargo, en otras intervenciones en el mundo 
real que hoy vivimos, algunas son muy valiosas y la ciencia no ha sido participe de ellas. Señala por ejemplo, la preservación de la biodiversidad posibilitada por las formas de conocimientos rurales e indígenas, las cuales se encuentran bajo amenazas por las intervenciones científicas.

\subsection{Acciones y políticas desde el Gobierno}

En este apartado se tomarán en cuenta las políticas y acciones emprendidas por el Gobierno Bolivariano desde la promulgación de la ley de aguas en el 2007.

Líneas generales relacionadas con el tema agua del Plan de Desarrollo Económico y Social de la Nación 2007- 2013: las estrategias y políticas para un modelo productivo socialista señala como propósitos: La biodiversidad nacional es una de las más altas del mundo y cuenta con abundantes recursos hídricos fuera de la zona norte costera. Ordenar el territorio asegurando la base de sustentación ecológica. Conservar las cuencas hidrográficas y la biodiversidad. Restringir las actividades en áreas de preservación. Reforzar las prácticas conservacionistas de los pueblos indígenas en sus territorios ancestrales. Manejar adecuadamente las Áreas Bajo Régimen de Administración Especial y demás áreas protegidas. Recuperar y mejorar los principales lagos y sus afluentes.

Líneas generales relacionadas con el tema agua del Plan de la Patria 2013-2019: los principales ejes de desarrollo ecosocialistas son: Protección de cuencas hidrográficas y ambientes naturales. Fortalecimiento y mejoras de los sistemas de agua potable. Consolidación del Plan Nacional de Aguas con la participación protagónica de los comités de agua y otras organizaciones del Poder Popular. Continuar incrementando y mejorando los sistemas de recolección y tratamiento de las aguas servidas en todo el territorio nacional.

Estas directrices se vienen a operacionalizar a través del ministerio que tiene la debida competencia para esta área como el Ministerio del Poder 
Popular para Ecosocialismo y Aguas; por medio del Plan Nacional de Gestión Integral de las Aguas. Según información de la página Web de este Ministerio (2016), una herramienta novedosa de este plan es el Tren Hídrico Nacional (THN), en donde los distintos componentes de la gestión integral del agua deben actuar de manera coordinada con los otros trenes productivos de la Nación.

Según la Asociación Venezolana para el Agua, AveAgua (ob.cit), el Plan Nacional de Recursos Hídricos tiene como misión formular, plantear y monitorear un conjunto de proyectos que satisfagan las necesidades actuales y futuras de abastecimiento de agua a la población, recuperación y saneamiento de las fuentes actualmente degradas y coadyuvar al ordenamiento territorial dentro de la Nueva Geopolítica Nacional.

Entre algunos proyectos y programas sectoriales que se han realizado según Aveagua (2011), cabe destacar: fortalecimiento de las organizaciones de productores con prácticas conservacionistas de siembra en curvas de nivel, agricultura en laderas, protección de suelos con barreras vegetales y protección de nacientes de agua. El proyecto denominado "Determinación de la Recarga y del Flujo de las Aguas Subterráneas", auspiciado por el Organismo Internacional de Energía Atómica (OIEA), que tiene como objetivo ofrecer una estrategia de aprovechamiento sustentable de las aguas subterráneas para los sistemas de riego. El Programa de Modernización del sistema de Pronóstico hidrometeorológico Nacional (VENEHMET), para el mejoramiento de la generación y difusión de la información hidrometeorológica. En cuanto al sector energético, puede identificarse el Plan de Manejo de la Cuenca del Río Caroní, cuenca de gran importancia estratégica para el país, ya que posee el mayor potencial hidroeléctrico de Venezuela y de América Latina.

Ahora bien, estas serían las políticas gubernamentales que en forma resumida se han venido implementando desde el año 2007 y que evidencian 
el esfuerzo del gobierno nacional por tener en cuenta la problemática de la escasez del agua en el país y tratar de darle una solución a través de la Gestión Integrada de Recursos Hídricos (GIRH).

Sin embargo, señala Aveagua (ob.cit), que si bien se observan diversas e importantes iniciativas en ese sentido, pareciera faltar coordinación entre ellas y sobre todo mayor participación de las ONGs y el sector académico en su diseño, instrumentación y evaluación. La coordinación de alto nivel y la voluntad política, dada la importancia de la integración de las múltiples visiones sectoriales, se presentan como elementos fundamentales de este proceso.

Asimismo, se requiere incorporar a la GIRH en todas las filas ministeriales relevantes, de forma tal que sus principios estén presentes en las diversas políticas, planes y programas de las diversas organizaciones públicas. Este diálogo debe involucrar a los usuarios, comunidades, gobiernos regionales, municipales, sector privado, organizaciones comunitarias y demás interesados, de manera de garantizar la coordinación de los esfuerzos en el manejo de las cuencas.

Por otro lado, el reciente informe de la Comisión Permanente de Ambiente, Recursos Naturales y Cambio Climático, de la Asamblea Nacional (2016), han señalado los siguientes aspectos con respecto a la problemática del agua como graves:

El Ministerio del Ambiente construyó trasvases de aguas negras que contaminaron las fuentes de agua potable de la Gran Caracas, el embalse de Camatagua de excelente calidad hasta el año 2009 es hoy un embalse contaminado. En el caso del Lago de Valencia se han construido obras para contaminar los embalses Pao / Cachinche y Pao / La Balsa a partir de las aguas negras de los ríos Maruria y Cabriales.

El Gobierno Nacional ha malgastado enormes montos de inversión en obras mal proyectadas, caso emblemático es el mayor acueducto del país: una 
tubería de $185 \mathrm{Km}$ que va del embalse de Matícora a la península de Paraguaná, este embalse está totalmente lleno de sedimentos y no garantiza ningún caudal. Obras inconclusas como el acueducto "Luisa Cáceres de Arismendi", en Margarita, para sustituir la antigua tubería construida en el año 1968. Esta tubería presenta enormes problemas de corrosión y roturas en el tramo submarino de $23 \mathrm{~km}$, con pérdidas de agua dulce.

Programa de Rehabilitación y Optimización de Plantas Potabilizadoras de Agua sin ejecutar. En el año 2011, Hidroven solicitó a la Corporación Andina de Fomento (CAF) recursos para diagnosticar el estado de operación de las siete (07) mayores plantas de agua potable del país. Este diagnóstico evidenció la escasa calidad de las aguas. El fenómeno natural El Niño, pese a ser un determinante climático en los efectos de la actual sequía, no es el causante de la crisis de agua a nivel nacional, es el mal manejo de las políticas públicas, la falta de planificación, la ineficiencia y abrupta corrupción.

El recurso hidráulico soporta el $60 \%$ de la generación eléctrica nacional, por lo tanto, es imprescindible obtener información de la verdadera situación del sistema eléctrico nacional y en especial del embalse Guri; se estima que la situación de escasez de agua se verá agravada. Finalmente, restituir el principio de descentralización establecido en el artículo 178 de la Constitución, que habilita a los municipios para la dotación y prestación de los servicios públicos domiciliarios, exhortando a la cooperación entre los distintos niveles de gobierno.

\section{Metodología}

Se trató de una investigación descriptiva, que "consiste en la caracterización de un hecho, fenómeno, individuo o grupo, con el fin de establecer su estructura o comportamiento" (Arias, 2006: 24). Se caracterizaron las funciones que han venido cumpliendo tanto el Estado, 
Nación y Gobierno en cuanto a la preservación y gestión de los recursos hídricos en el país, para generar una nueva visión geopolítica de los mismos.

En cuanto al diseño, fue una investigación documental, cuyo proceso está basado "en la búsqueda, recuperación, análisis, crítica e interpretación de datos secundarios" (Arias, ob.cit: 27). Se realizó una búsqueda y recopilación de diferentes fuentes (impresas y electrónicas), normativa legal e institucional relacionada con el tema. Se plasmaron las críticas e interpretaciones de las mismas a través del análisis de contenido para concluir con los aspectos más resaltantes en el rol del Estado, Nación y Gobierno.

\section{Conclusiones}

En Venezuela se ha desarrollado todo un entramado institucional, jurídico y gubernamental con el propósito de atender la gestión integral de los recursos hídricos, dado los problemas de escasez a nivel mundial, regional y local; con el fin de generar una nueva visión geopolítica de los recursos hídricos. Cada ámbito por separado ha tratado de abordar dicha situación, sin embargo, el esfuerzo no ha dado hasta ahora los mejores resultados y ante la impostergable medida para iniciar con las posibles soluciones, se hace necesario establecer transformaciones en las funciones que les corresponde tanto al Estado, la nación y el gobierno.

Desde la percepción De Lisio (2008), con la ecopolítica del Estadonación se requiere de: un Estado central que conoce las amenazas ambientales, propicia la interrelación entre los acuerdos internacionales y regionales para coordinar políticas con los gobiernos locales. Es decir, incorpora la seguridad ambiental local en el proyecto nacional. Un gobierno local que conoce sus amenazas y oportunidades ambientales; propicia la reducción de la vulnerabilidad ambiental local estimulando el aprovechamiento sostenible de los recursos naturales locales mediante la aplicación del conocimiento propio. Esto es lo que se requiere en Venezuela, la interrelación 
entre el gobierno nacional y los gobiernos locales indistintamente de posiciones políticas e ideológicas, así como la transferencia de competencias a los municipios según el artículo 178 de la Constitución.

Por su parte la Red ARA (2011), sostiene que se debe exigir al gobierno nacional cumplir con la legislación existente en materia de la gestión del recurso agua. Fortalecer a las instituciones encargadas de la gestión del agua. Actualizar las normativas y los planes de manejo relacionados con las cuencas hidrográficas. Específicamente, elaborar el reglamento de la Ley de Aguas con la participación de diferentes actores y usuarios, ya que tiene 5 años de mora.

Desarrollar canales de coordinación, cooperación e intercambio de información entre los principales usuarios del recurso, el gobierno nacional y los gobiernos regionales y locales, así como con las universidades, las ONG y el sector privado, para compartir la responsabilidad de promover la defensa, conservación y mejoramiento del recurso hídrico a nivel nacional, sin exclusión. Incorporar la adaptación al cambio climático como elemento estratégico en la gestión integral de recursos hídricos.

Se han dado pasos importantes en lo que se refiere a las funciones del Estado venezolano en los ámbitos jurídicos, institucionales y hasta en el incremento del acceso al agua potable, permitiendo a Venezuela cumplir algunas de las metas del milenio, pero aún quedan pendientes aspectos fundamentales de la Gestión Integral de los Recursos Hídricos que deben ser atendidos.

Según señala AveAgua (ob.cit); en cuanto al sentimiento nacional, de cultura y responsabilidad por una conciencia ecologista, que lleve a la preservación de los recursos hídricos en Venezuela. Es fundamental estimular el desarrollo de una conciencia colectiva sobre el uso racional de este recurso, y educar a la ciudadanía hacia un uso sustentable del agua. Son muchos los estados en el país que mantienen patrones de consumo que superan, duplican y triplican la dotación sugerida por organismos internacionales. Es importante 
informar a la ciudadanía en torno a los escenarios desfavorables sobre posibles impactos del cambio climático, así como problemas que ya hoy se tienen en algunas regiones por degradación de los suelos y desertificación de ciertas zonas.

En materia del gobierno, Vitalis (ob.cit), recomienda al poder ejecutivo la creación del Consejo Nacional del Ambiente, que integre a los organismos públicos, privados y sociedad civil. Así como, promover la cooperación entre el gobierno y los municipios para atender las problemáticas del recurso hídrico.

\section{Referencias}

Arias, F. (2006). El proyecto de investigación: introducción a la metodología científica. (5taed). Caracas: Episteme.

Asamblea Nacional (2016). Comisión permanente de ambiente recursos naturales y cambio climático. Primer informe problemática del agua. Caracas.

Asociación Venezolana para el Agua (AveAgua) (2011). Situación de los Recursos Hídricos en Venezuela. / Eds. Zoyla Martínez \& Diego Díaz Martin. -2da. Ed.- Caracas.

De Lisio, A. (2008). La seguridad ambiental venezolana bajo la concepción eco-política del Estado multidimensional. Caracas. Instituto Latinoamericano de Investigaciones Sociales.

De Sousa, B. (2010). Descolonizar el saber, reinventar el poder. Montevideo (Uruguay). Ediciones Trilce.

Ley de Aguas. Gaceta Oficial № $\mathbf{3 8 . 5 9 5}$ del 02/01/2007. Caracas, Venezuela. Ministerio del Ambiente (1998). Intervención de Venezuela. Conferencia Internacional sobre Agua y Desarrollo Sustentable. Paris, Francia. Morin, E. (2011). La vía para el futuro de la humanidad. Barcelona (España). Paidos. 
Ministerio del Poder Popular para Ecosocialismo y Aguas (2016). [Página Web en línea].

Quagliotti, B. (2011). El agua recurso vital de las civilizaciones. [Documento en línea]. Recuperado de:

http://www.gestiopolis.com/el-agua-recurso-vital-de-las-civilizaciones/

Red ARA (2011). Aportes para un diagnóstico de la problemática ambiental de Venezuela: La visión de la Red ARA. Caracas, Venezuela. Recuperado de:

https://de.slideshare.net/ONGVitalis/situacin-ambiental-de-venezuela$\underline{2013}$

República Bolivariana de Venezuela (2016). Plan de Desarrollo Económico y Social de la Nación 2007- 2013. Recuperado de:

http://www.mppp.gob.ve/wp-content/uploads/2013/09/Plan-de-laNación-2007-2013.pdf

República Bolivariana de Venezuela (2016). Plan de la Patria 2013-2019. Recuperado de:

http://gobiernoenlinea.gob.ve/home/archivos/PLAN-DE-LA-PATRIA2013-2019.pdf

Rojas, R. (2011). Fiesta, imaginario político y nación. San Felipe, Venezuela. Edición de la Universidad Nacional Experimental del Yaracuy (UNEY).

VITALIS (2013). Situación Ambiental de Venezuela 2012. Análisis de Percepción del Sector. Editores y Compiladores: D. Díaz Martín, Y. Frontado, M. Da Silva, A. Lizaraz, I. Lameda, V. Valera, C. Gómez. E. Monroy, Z. Martínez, J. Apostólico y G. Suárez. Recuperado de: http://www.ucv.ve/fileadmin/user upload/cenamb/Situacion-Ambientalde-Venezuela-2012.pdf 


\section{Carlos Javier Lizcano Chapeta}

e-mail: lizcha 4@hotmail.com

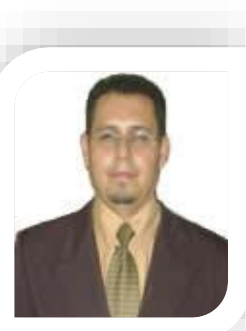

Licenciado en Educación, Abogado, Magister en Ciencias Políticas y actualmente Doctorando en Estudios Políticos de la Universidad de Los Andes. Profesor contratado de la Universidad Pedagógica Experimental Libertador (UPEL), Núcleo Mérida. Profesor invitado del Centro de Estudios Políticos y Sociales de América Latina (CEPSAL) en la Universidad de Los Andes (ULA). Investigador reconocido por el Programa de Estímulo al Investigador (PEI), adscrito al Grupo de Investigación de Geopolítica del Ambiente y Relaciones Internacionales (GIGARI) de la Universidad de Los Andes (ULA) y del Núcleo de Investigación Educativa Mérida de la UPEL. Ha impartido las asignaturas de Seminario de Investigación en los Postgrados de la UPEL y la ULA. Se ha desempeñado como tutor de tesis. 